\title{
Effects of Various Cooking Methods on Content, Oxygen Radical Absorbance Capacity, and Bioaccessibility of Caffeoylquinic Acids in Ligularia fischeri (Ledeb.) Turcz, Gom-Chi
}

\author{
Yu-Ra Son ${ }^{1} \&$ Soon-Mi Shim ${ }^{1}$ \\ ${ }^{1}$ Department of Food Science and Technology, Sejong University, Gunja-dong, Seoul, Republic of Korea \\ Correspondence: Soon-Mi shim, Department of Food Science and Technology, Sejong University, 98 Gunja-dong, \\ Seoul 143-747, Republic of Korea. Tel: 82-2-3408-3229. E-mail: soonmishim@sejong.ac.kr
}

Received: April 12, 2014 Accepted: May 21, 2014 Online Published: July 15, 2014

doi:10.5539/jas.v6n8p19 URL: http://dx.doi.org/10.5539/jas.v6n8p19

\begin{abstract}
We hypothesized that cooking methods generally applied to Gom-chi preparation such as blanching, pan frying, and microwaving could change content, oxygen radical absorbance capacity (ORAC), and bioaccessibility of caffeoylquinic acids (CQAs). Mono-, di-, and tri-CQAs were identified and quantified by using an ultra-performance liquid chromatograph-photodiode array detector. In vitro biomimick system was performed for assessing the bioaccessibility of CQAs. The free radical scavenging capacity was measured by ORAC assay, expressed as trolox equivalents (TE). The amount of 5-CQA, 4-CQA, 3-CQA, and 3,4-di-CQA were 136.72, $2144.44,16.81$, and $421.93 \mu \mathrm{g}$, respectively, in $\mathrm{g}$ of fresh Gom-chi. The highest amount of CQAs and TE was observed in microwaving for $3 \mathrm{~min}$ and pan frying for $3 \mathrm{~min}$, respectively. The ranking of total CQAs were found in the order of microwaving $3 \mathrm{~min}(239.96 \mu \mathrm{g} / \mathrm{g})>$ microwaving for $2 \mathrm{~min}(206.11 \mu \mathrm{g} / \mathrm{g})>$ blanching for 3 $\min (191.94 \mu \mathrm{g} / \mathrm{g})>$ blanching for $5 \min (180.32 \mu \mathrm{g} / \mathrm{g})>$ pan for frying $5 \mathrm{~min}(161.20 \mu \mathrm{g} / \mathrm{g})>$ pan frying for 3 $\min (115.83 \mu \mathrm{g} / \mathrm{g})$. The bioaccessibility of total CQAs were $16.42,17.64,14.39,13.29$, and $12.43(\%)$ in fresh, blanching for $3 \mathrm{~min}$, blanching for $5 \mathrm{~min}$, pan frying for $3 \mathrm{~min}$, and pan frying for $5 \mathrm{~min}$, respectively. Among the cooking methods, blanching for $3 \mathrm{~min}$ had the highest amount of CQAs after digestion. Our results suggest that blanching 3 min could be a suggested optimal cooking method for Gom-chi to improve CQAs absorption.
\end{abstract}

Keywords: Ligularia fischeri (Ledeb) Turcz, caffeoylquinic acids (CQAs), Oxygen Radical Absorbance Capacity (ORAC), bioaccessibility

\section{Introduction}

Ligularia fischeri (Ledeb) Turcz known as 'Gom-chi' in Korea, a member of the compositae, is stably supplied and mostly consumed in Korea (Shang et al., 2010). It is also a perennial vegetable plant and cultivated in moist, shady areas in various countries in Europe and eastern Korea (Shang et al., 2010; Kim et al., 2012). Gom-chi has been recognized as plant predominantly contains caffoylquinic acids (Kim et al., 2010). Caffeoylquinic acids (CQAs), as a generally known group of antioxidant compounds, are formed by caffeoyl groups linked to quinic acid (Kim et al., 2012). The categorization of CQAs is classified by both the structural position and the number of caffeoyl groups bound to quinic acid (Figure 1); mono-caffeoylquinic acids (mono-CQAs), di-caffeoylquinic acids (di-CQAs) and tri-caffeoylquinic acids (tri-CQAs). Among them, mono-CQAs and di-CQAs are commonly found in plants (Lee et al., 2010). These are known to provide diverse biological functions such as a scavenging of free radicals, reacting to enzymatic browning, preventing fungal pathogens, and resisting pathogenic insects (Kim et al., 2012). Owing to various functions of CQAs, Gom-chi is known to contain a significant amount of CQAs and has been used as a traditional medicine for the treatment of jaundice, scarlet fever, rheumatoid arthritis, and liver injury (Shang et al., 2010). 


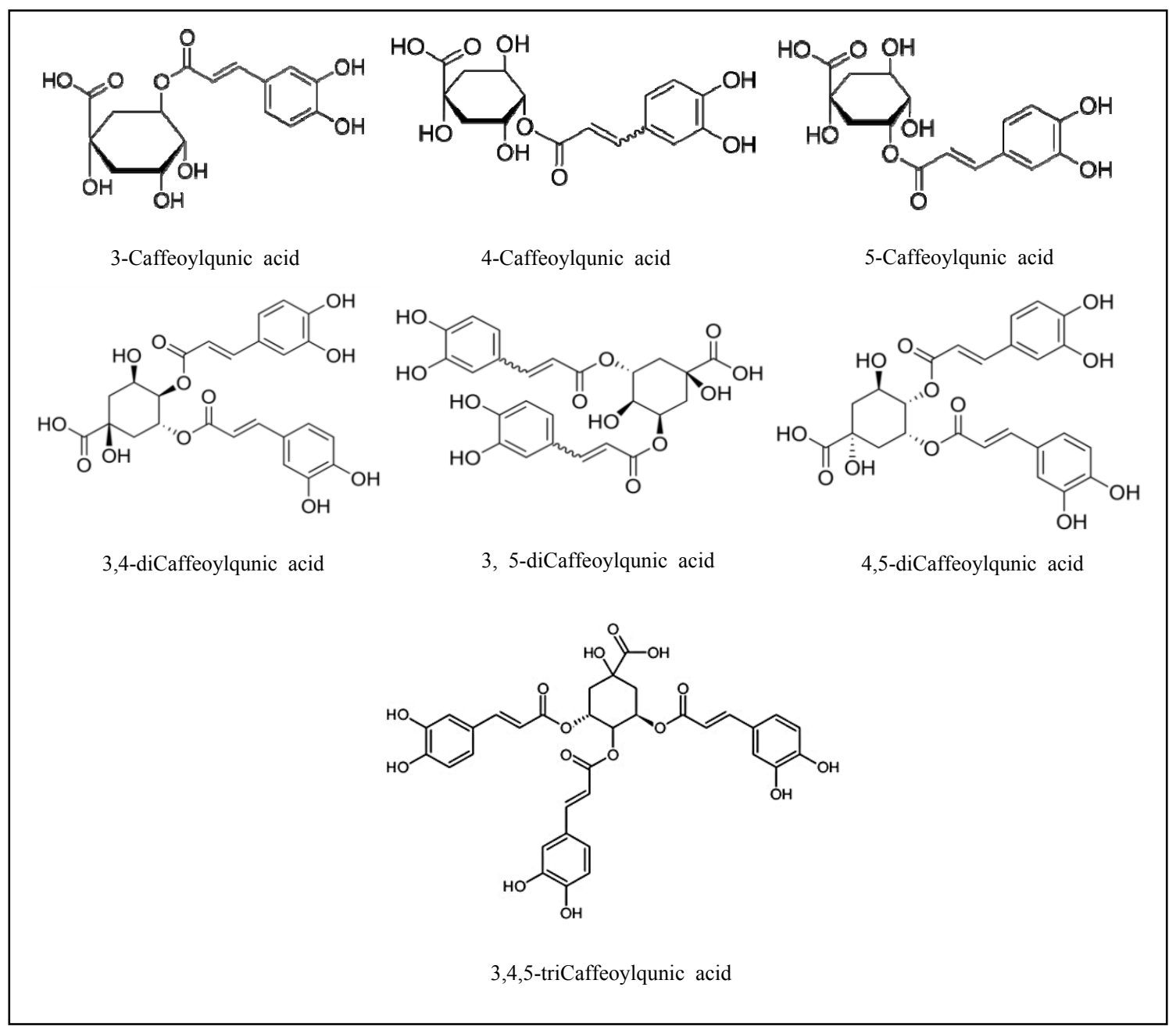

Figure 1. Structures of the caffeoylquinic acids (CQAs)

As consumption of Gom-chi has increased in recent years, many easy cooking methods including blanching, pan frying, and microwaving has been introduced (Pellegrini et al., 2010). One previous study suggested that various cooking preparations could affect the polyphenol content in vegetables (Miglio et al., 2007). For instance, the study found that total phenol compounds in raw carrots were $69.6 \mathrm{mg} / 100 \mathrm{~g}$ of dry weight, whereas steamed and fired carrots were 39.6 and $48 \mathrm{mg} / 100 \mathrm{~g}$ of dry weight, respectively. Gom-chi, a representative leafy vegetable is generally consumed by various cooking methods such as blanching, pan frying, and microwaving. However, there is limited research on the effects of cooking methods on their antioxidant capacity, and on the bioaccessibility referred the fraction of released food elements in the human gut that becomes available for absorption into human body (Yang et al., 2012; Lee et al., 2013). Thus, the objective of the current study was to determine the CQA content in Gom-chi according to various cooking methods, to estimate bioaccessibility of CQAs, and to measure the antioxidant capacity by oxygen radical absorbance capacity (ORAC) assay.

\section{Materials and Methods}

\subsection{Standards and Chemical Reagents}

CQAs such as 3-caffeoylquinic acid (chlorogenic acid), 5-caffeoylquinic acid (neochlorogenic acid), 4-caffeoylquinic acid, 3,4-di-caffeoylquinic acid, 6-hydroxy-2,5,7,8-tetramethylchromane-2-carboxylic acid (trolox), digestive enzymes such as amylase, porcine pepsin, pancreatin, lipase, and bile acid, potassium phosphate dibasic (98\%) and potassium phosphate monobasic (98\%), 2,2'-Azobis(2-methylpropion amidine) dihydrochloride (AAPH) and fluorescein stock solution were purchased from Sigma-Aldrich (St. Louis, Mo, USA). Solvents for ultra-performance liquid chromatography (UPLC) grade of ethanol, methanol, and water were purchased from J.T. Baker (Phillipsburg, Nj, USA) and formic acid was obtained from Fisher Scientific (Pittsburgh, Pa, USA). Ascorbic acid and sodium bicarbonate were purchased from Junsei-Chemical (Tokyo, 
Japan) and Fluka (Buchs, Switzerland), respectively. Hydrochloric acid and sodium hydroxide were obtained from Dae-Jung Chemical (Kyung-gi, Korea) and Duksan (Kyung-gi, Korea), respectively. All other chemicals and solvents used were of analytical grade.

\subsection{Sample Preparation}

Gom-chi was harvested from Buyeo-gun, Chungcheongnam-do, Republic of Korea in February 2013, and it was washed with deionized water. An aliquot amount of lipholized sample ( $7 \mathrm{~g}$ ) was mixed with $350 \mathrm{~mL}$ of $50 \%$ ethanol and then sonicated for $40 \mathrm{~min}$ at room temperature. For cooking, aliquots $(800 \mathrm{~g}$ and $500 \mathrm{~g})$ of fresh sample were mixed with each $8 \mathrm{~L}$ and $5 \mathrm{~L}$ water, respectively, and then it was blanched for both 3 and $5 \mathrm{~min}$. Pan fried Gom-chi was cooked with $50 \mathrm{~mL}$ of cooking oil for both 3 and $5 \mathrm{~min}$. Gom-chi was cooked in the microwave at a power of $700 \mathrm{~W}$ for both 2 and $3 \mathrm{~min}$ for the microwaving sample.

\subsection{Identification and Quantification of CQAs}

The aliquot amount of sample $(0.02 \mathrm{~g})$ mixed with methanol $(1 \mathrm{~mL})$ was sonicated at room temperature for 60 min and then filtered through a $0.45 \mu \mathrm{m}$ of PVDF syringe filter (Whatman Ltd, Piscataway, $\mathrm{Nj}$, USA). CQAs in Gom-chi were quantified and identified by ultra performance liquid chromatography (UPLC) equipped with a photodiode array (PDA) detector, Accela auto sampler, and Accela 600 pump (Thermo Scientific, Vantaa, Finland). The analytical condition was slightly modified from method described in a previous study(Kim et al., 2012). The separation of CQAs was performed on a Hypersil GOLD column $(50 \times 2.1 \mathrm{~mm}, 1.9 \mu \mathrm{m}$, Thermo Scientific) in the mobile phase of A $(0.1 \%$ formic acid in $100 \%$ methanol) and B $(0.1 \%$ formic acid in water). The gradient of the mobile phase system was used as follows: $0-5 \mathrm{~min}, 10-15 \%$ of A; 5-6.88 min, $15-90 \%$ of A; 6.88-6.9 $\mathrm{min}, 90-10 \%$ of A; 6.9-9 min, $10-10 \%$ of A. An injection volume was $2 \mu \mathrm{L}$. The sample tray temperature was set to $4{ }^{\circ} \mathrm{C}$, and PDA detector wavelength was fixed at $280 \mathrm{~nm}$ and $330 \mathrm{~nm}$.

\subsection{Bioaccessibility of CQAs}

The in vitro gastrointestinal digestion (GI) model system, which simulates the human upper digestive tract including salivary, gastric and small intestinal phases, was used according to the previous method (Shim et al., 2012). Each sample was suspended in aliquot of $20 \mathrm{mM}$ phosphate buffer. $0.5 \%$ ascorbic acid was added in order to prevent oxidation. For the salivary simulation, amylase $(0.2 \mathrm{mg} / \mathrm{mL}$ in $20 \mathrm{mM}$ phosphate buffer) was added and the initial $\mathrm{pH}$ was adjusted to $\mathrm{pH} 6.9$ by $20 \mathrm{mM}$ phosphate buffer. Samples were incubated in a shaking water bath at $37^{\circ} \mathrm{C}$ with $100 \mathrm{rpm}$ for $3 \mathrm{~min}$. The gastric phase was carried out by the addition of porcine pepsin $\left(3 \mathrm{mg} / \mathrm{mL}\right.$ in $100 \mathrm{mM} \mathrm{NaHCO}_{3}$ ) and the acidification of the samples to $\mathrm{pH} 2$ with $0.1 \mathrm{M} \mathrm{HCl}$. The samples were allowed to incubate for $30 \mathrm{~min}$ in a shaking water bath $\left(37^{\circ} \mathrm{C}\right.$ and $\left.100 \mathrm{rpm}\right)$. For the intestinal phase, $100 \mathrm{mM}$ sodium bicarbonate solution was added to neutralize the samples to $\mathrm{pH}$ 5.3. A pancreatic enzyme mixture $(0.4$ $\mathrm{mg} / \mathrm{mL}$ of pancreatin in $20 \mathrm{mM}$ phosphate buffer, $0.2 \mathrm{mg} / \mathrm{mL}$ of lipase in $20 \mathrm{mM}$ phosphate buffer and 2.4 $\mathrm{mg} / \mathrm{mL}$ of bile acid in $20 \mathrm{mM}$ phosphate buffer) was added. The final $\mathrm{pH}$ was adjusted to $\mathrm{pH} 6$ with $0.1 \mathrm{M}$ $\mathrm{NaOH}$ and then incubated in a shaking water bath for $60 \mathrm{~min}$ at $37^{\circ} \mathrm{C}$ and speed of $100 \mathrm{rpm}$. The final volume of all samples was equalized to $10 \mathrm{~mL}$ of the volume by using a $20 \mathrm{mM}$ phosphate buffer. Finally, the aliquots from digesta were centrifuged at $4{ }^{\circ} \mathrm{C}$ and $3000 \mathrm{rpm}$ for $30 \mathrm{~min}$ and then the supernatant was prepared for UPLC analysis.

\subsection{Measurement of Antioxidant Capacity}

The oxygen radical absorbance capacity (ORAC) assay was used to measure the antioxidant capacity of Gom-chi according to Huang et al., (2002). In this assay, a $150 \mu \mathrm{L}$ of $10 \mathrm{nM}$ fluorescein stock solution in a phosphate buffer solution ( $\mathrm{pH} 7.4)$, and $25 \mu \mathrm{L}$ of sample or trolox as standard were carried out in 96-well plates. After

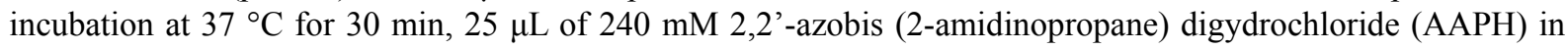
PBS was added. The fluorescence was measured using a micro plate reader (Varioskan Flash, Thermo Scientific, USA) and read every 10 minutes, 10 times in total, at $485 \mathrm{~nm}$ for excitation and $520 \mathrm{~nm}$ for emission. The results were calculated using the distinction of the areas under fluorescein decay curve between the sample and the blank according to the following formula:

$$
\text { ORAC }(\mu \mathrm{M} \text { of trolox equivalents })=\left\{\mathrm{C}_{\text {Trolox }} \times\left(\mathrm{AUC}_{\text {Sample }}-\mathrm{AUC}_{\mathrm{Blank}}\right) \times \kappa\right\} /\left(\mathrm{AUC}_{\text {Trolox }}-\mathrm{AUC}_{\mathrm{Blank}}\right)
$$

Where: $\mathrm{C}_{\text {Trolox }}$ - concentration of trolox; $\mathrm{AUC}$ - the area under the curve; $\kappa$ - dilution factor.

\subsection{Statistical Analysis}

Results were presented as a mean \pm standard deviation (SD) from triplicate sets of each experiment. Analysis of variance (ANOVA), Tukey's post hoc test and correlation analysis were performed to evaluate significant differences among groups at the significant level of 5\% by using Graphpad Prism 3.0 software (Graphpad, San 
Diego, Ca, USA).

\section{Results and Discussion}

\subsection{Simultaneous Quantification and Identification of CQAs From Gom-Chi}

An LC chromatogram of CQAs from standard mixtures of 5-CQA, 4-CQA, 3-CQA, 3,4-di-CQA is shown in Figure 2A. CQAs were simultaneously identified with a modified method from a previous study (Kim et al., 2012), adjusting the mobile phase to weak elution by replacing $0.1 \%$ trifluoracetic acid (TFA) in water and ACN with $0.1 \%$ formic acid in water and $0.1 \%$ formic acid in methanol. Figure $2 \mathrm{~B}$ presents the LC chromatogram of CQAs from 50\% ethanol extract of fresh Gom-chi. Fresh Gom-chi was observed to consist of four CQAs;5-CQA 4-CQA, 3-CQA, and 3,4-di-CQA. The CQAs were identified as 5-CQA, 4-CQA, 3-CQA, and 3,4-di-CQA according to matching retention time of its standard, and they were quantified by the standard curve of each standard (Figure 2A). The amount of 5-CQA, 4-CQA, 3-CQA, and 3,4-di-CQA was 136.72, 2144.44, 16.81, and $421.93 \mu \mathrm{g} / \mathrm{g}$ fresh weight of fresh Gom-chi, respectively. Different constituents of CQAs in fresh Gom-chi were previously identified by other investigators (Shang et al., 2010; Kim et al., 2012). Kim et al. (Kim et al., 2012) reported that methanol extract of air dried Gom-chi contained 5-CQA, 3,4-di-CQA, 3,5-di-CQA, and 4,5-di-CQA. Shang et al., (Shang et al., 2010) found that four major components including 5-CQA, 3,4-di-CQA, 3,5-di-CQA, and 4,5-di-CQA were identified from 70\% ethanol extract of air-dried Gom-chi. Compared to the results of our study, those studies detected several di-CQA including 3,4-di-CQA, 3,5-di-CQA, and 4,5-di-CQA rather than others in air-dried Gom-chi. This implies that variances of CQAs profile in Gom-chi may be due to differences in origins, extract solvents, and drying methods.
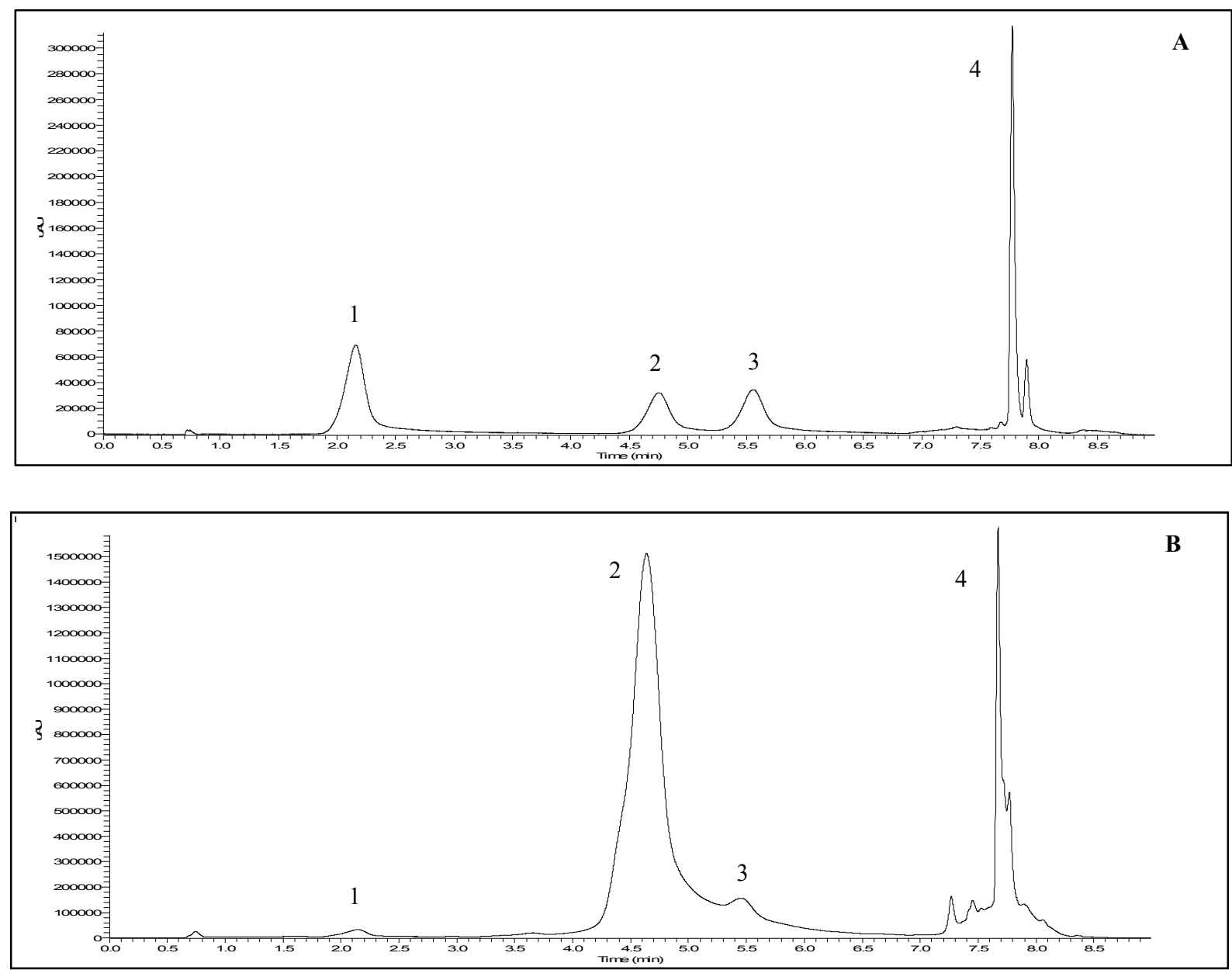

Figure 2. Ultra performance liquid chromatography - photodiode array (UPLC-PDA) chromatogram of standard mixtures of each CQA (A) and CQAs from 50\% ethanol extract of fresh Gom-chi (B) (Peak 1:5-CQA, Peak 2 : 4-CQA, Peak 3 : 3-CQA, Peak 4: 3,4-diCQA) 


\subsection{Effect of Various Cooking Methods on Content of CQAs From Gom-Chi}

The effect of various cooking methods on CQA content in Gom-chi is shown in Table 1. Fresh Gom-chi was observed to contain total CQAs of $2710.9 \mu \mathrm{g} / \mathrm{g}$ fresh weight. The highest amount of 4-CQA (2144.44 $\mu \mathrm{g} / \mathrm{g}$ fresh weight) was observed, followed by 3,4-di-CQA (412.93 $\mu \mathrm{g} / \mathrm{g}$ fresh weight), 5-CQA (136.72 $\mu \mathrm{g} / \mathrm{g}$ fresh weight), and 3-CQA (16.81 $\mu \mathrm{g} / \mathrm{g}$ fresh weight). The total amount of CQAs was significantly reduced after cooking procedures compared to fresh Gom-chi $(\mathrm{p}<0.05)$. The rankings of total CQAs in different cooking methods were microwaving for $3 \mathrm{~min}>$ microwaving for $2 \mathrm{~min}>$ blanching for $3 \mathrm{~min}>$ blanching for $5 \mathrm{~min}>$ pan frying for $5 \mathrm{~min}>$ pan frying for $3 \mathrm{~min}$. In the same cooking procedure, the amount of 5-CQA and 3-CQA were greatly enhanced as the cooking time increased. The current study found that pan frying for $3 \mathrm{~min}$ had the greatest loss of total CQAs while microwaving for 3 min showed the highest amount of CQAs. The microwave oven is set heat food, using 2,500 megahertz of radio wave. When micro waves are absorbed into all food by water, sugars, and fats, it can move water molecules. The highest concentration of CQAs could be due to this process; evaporating of water in Gom-chi, resulting in concentrating CQAs in Gom-chi. A similar pattern to results from current the study was reported by a previous study, indicating that the amount of 3-CQA in carrots decreased by various cooking preparations including boiling, steaming, and frying (Miglio et al., 2007). The study found 2.2 and $3.1 \mathrm{mg} / 100 \mathrm{~g}$ of dry weight of 3-CQA in each steamed and fried carrot, respectively. The study also found that boiling preparations had the highest detrimental consequence on 3-CQA of carrots, due to their diffusion followed by the breaking of cellular components in boiling water. Farah et al., (Farah et al., 2005) reported that the amount of total 3-CQA and 3-CQA lactones from coffee decreased to less than $5.2 \%$ and $20 \%$, respectively, of their maximal values at a longer time of roasting. Comparable findings reported that the total phenol content in various vegetables was shown to have a decreasing tendency after boiling (Natella et al., 2010).

Table 1. Effect of various cooking methods on CQAs contents from Gom-chi

\begin{tabular}{|c|c|c|c|c|c|}
\hline \multirow{2}{*}{ Cooking methods } & \multicolumn{5}{|c|}{ Amount of CQAs ( $\mu \mathrm{g} / \mathrm{g}$ fresh weight) } \\
\hline & 5-CQA & 4-CQA & 3-CQA & 3,4-di-CQA & Total CQAs \\
\hline Fresh & $136.72 \pm 0.68$ & $2144.44 \pm 121.92$ & $16.81 \pm 0.66$ & $412.93 \pm 7.23$ & $2710.9 \pm 126.55^{\mathrm{a}}$ \\
\hline $\begin{array}{l}\text { Blanching } \\
3 \text { min }\end{array}$ & $11.38 \pm 0.11$ & $122.66 \pm 0.05$ & $2.23 \pm 0.06$ & $55.68 \pm 0.43$ & $191.94 \pm 0.56^{\mathrm{c}}$ \\
\hline $\begin{array}{c}\text { Blanching } \\
5 \mathrm{~min}\end{array}$ & $26.97 \pm 0.80$ & $117.48 \pm 6.97$ & $4.37 \pm 0.79$ & $31.5 \pm 3.61$ & $180.32 \pm 10.36^{\mathrm{c}}$ \\
\hline $\begin{array}{c}\text { Pan frying } \\
3 \text { min }\end{array}$ & $9.40 \pm 0.99$ & $79.69 \pm 2.18$ & $2.41 \pm 0.27$ & $24.33 \pm 1.13$ & $115.83 \pm 3.96^{\mathrm{e}}$ \\
\hline $\begin{array}{c}\text { Pan frying } \\
5 \text { min }\end{array}$ & $13.78 \pm 1.21$ & $80.92 \pm 3.47$ & $4.56 \pm 0.29$ & $61.94 \pm 13.08$ & $161.20 \pm 12.23^{\mathrm{d}}$ \\
\hline $\begin{array}{l}\text { Microwaving } \\
2 \mathrm{~min}\end{array}$ & $8.97 \pm 0.77$ & $158.01 \pm 15.96$ & $2.98 \pm 0.14$ & $36.14 \pm 1.60$ & $206.11 \pm 16.72^{\mathrm{c}}$ \\
\hline $\begin{array}{c}\text { Microwaving } \\
3 \mathrm{~min}\end{array}$ & $10.82 \pm 1.57$ & $175.90 \pm 6.92$ & $4.12 \pm 0.23$ & $49.13 \pm 1.94$ & $239.96 \pm 4.14^{\mathrm{b}}$ \\
\hline
\end{tabular}

*Different letters indicate a significant difference among cooking methods at $\mathrm{p}<0.05$.

\subsection{Effects of Various Cooking Methods on Oxygen Radical Absorbance Capacity From Gom-Chi}

Oxygen radical absorbance capacity (ORAC) of Gom-chi prepared from various cooking procedures was measured by ORAC assay (Figure 3). It was expressed as Trolox equivalents (TE, $\mu \mathrm{mol} \mathrm{TE} / 100 \mathrm{~g}$ ), representing a total antioxidant capacity measured by time. The ORAC value of fresh Gom-chi was $12.69 \mu \mathrm{mol} \mathrm{TE} / 100 \mathrm{~g}$. Fresh Gom-chi showed the highest TE $(12.69 \mu \mathrm{mol} \mathrm{TE} / 100 \mathrm{~g})$ followed in the order of pan frying for $3 \mathrm{~min}$ $(9.88 \mu \mathrm{mol} \mathrm{TE} / 100 \mathrm{~g})$, microwaving for $3 \mathrm{~min}(9.85 \mu \mathrm{mol} \mathrm{TE} / 100 \mathrm{~g})$, pan frying for $5 \mathrm{~min}(9.84 \mu \mathrm{mol} \mathrm{TE} / 100 \mathrm{~g})$, blanching for $3 \mathrm{~min}(9.69 \mu \mathrm{mol} \mathrm{TE} / 100 \mathrm{~g})$, microwaving for $2 \mathrm{~min}(9.49 \mu \mathrm{mol} \mathrm{TE} / 100 \mathrm{~g})$, blanching for $5 \mathrm{~min}$ $(9.17 \mu \mathrm{mol} \mathrm{TE} / 100 \mathrm{~g})$. The ORAC value was not directly proportional to the amount of total CQAs from Gom-chi. A previous study (Delonga \& Mazor, 2009) reported that when cocoa liquor samples had a greater 
amount of total polyphenols, the ORAC value ( $\mu$ mol TE/g of defatted cocoa liquors) was higher. In order to establish a relationship between the content of CQAs and the antioxidant activity, a correlation analysis was conducted. A significant correlation $\left(\mathrm{r}^{2}\right)$ was found between the total CQAs content and TE in Gom-chi blanched for $3 \mathrm{~min}$ (0.98), blanched for $5 \mathrm{~min}(0.99)$, and pan fried for $3 \mathrm{~min}(0.95)$.

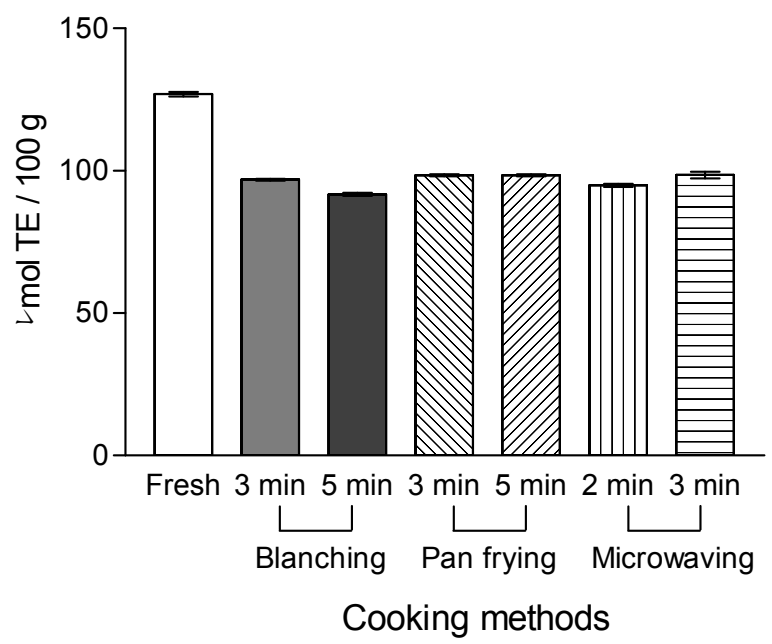

Figure 3. Effect of cooking methods on antioxidant capacity from Gom-chi. *Different letters indicate significant difference among cooking methods at $\mathrm{p}<0.05$

\subsection{Effects of Various Cooking Methods on Digestive Recovery of CQAs From Gom-Chi}

The relatively recovered amounts of CQA in Gom-chi after in vitro digestion in accordance to various cooking methods are shown in Table 2. Regardless of the cooking method, among the CQAs, the highest amount of measured 4-CQA ranged from 10.89 to $344.67 \mu \mathrm{g} / \mathrm{g}$ fresh weight, while the least amount of 3-CQA obtained ranged from 0.61 to $14.85 \mu \mathrm{g} / \mathrm{g}$ fresh weight. The ranking of the recovered total CQAs was fresh Gom-chi $>$ blanching for $3 \mathrm{~min}>$ blanching for $5 \mathrm{~min}>$ pan frying $5 \mathrm{~min}>$ pan frying $3 \mathrm{~min}$. A previous study observed 38 $\%$ of CQAs in coffee beverage recovered after digestion (Tagliazucchi et al., 2012). Interestingly, total CQAs in microwaved Gom-chi was not detected, indicating that the CQAs were barely digestible. In the same way, microwaving decreases the metabolic behavior or alters food elements, resulting in disorders in digestive system (Koop. 2000). The study found that digestibility of microwaved fruits and vegetables was significantly lowered. Likewise, less various CQA in Gom-chi were released in digesta because CQAs in microwaved Gom-chi were less digested by interrupting digestive system by microwaving. The bioaccessibility of total CQAs (\%) in Gom-chi according to various cooking methods ranged from 12.43 to $17.64 \%$ (Table 2). Among CQAs in fresh Gom-chi, 3-CQA was recovered the most after the in vitro digestion model system (88.34\%) followed by 5-CQA (24.41\%), 4-CQA (16.07\%), and 3,4-di-CQA (12.58\%). The bioaccessibility of total CQAs was significantly greater in Gom-chi blanched for 3 min than fresh and other cooked one $(p<0.05)$. As the cooking time increased, the bioaccessibility of total CQAs were reduced 0.82 and 0.94 times in blanching and pan frying, respectively. However, the bioaccessibility of total CQAs in pan frying for 3 min Gom-chi was slightly but not significantly higher than pan frying for $5 \mathrm{~min}$. To our knowledge, there is limited research on the bioaccessibility and absorption of various CQAs in humans. Previous studies that were done on the stability of 3-CQA measured the digestive recovery of this compound after incubation with human gastric juice (Olthof et al., 2001). The stability of 3-CQA expressed as the percentage was $101 \%$ and $99 \%$ after 30 min and 120 min incubated with human gastric juice, respectively. This indicated that 3-CQA was more stable in human digestive system. Therefore the bioaccessibility of 3-CQA from fresh Gom-chi was higher than other CQAs. Another study observed that bioaccessibility of total CQAs in 50\% coffee in water was approximately 65\% (Tagliazucchi et al., 2012). Compared to current study, CQAs from coffee had greater bioaccessiblilty than that from Gom-chi. The decrease in CQAs bioaccessibility from Gom-chi after digestion may be due to degradation. A previous study reported that a general degradation of 3-CQA took place during the in vitro digestion process of capers and sea fennel (Tagliazucchi et al., 2012). Also, Hornero-Méndez et al., (Hornero-Méndez \& Mínguez-Mosquera, 2007) revealed that the bioaccessibility of carrot crude containing the carotenoids was reduced from $27 \%$ to $13 \%$ after heating at $100{ }^{\circ} \mathrm{C}$ in a water bath for $15 \mathrm{~min}$. Consequently we might conclude that cooking procedures affect the 
bioaccessibility of CQAs from the food matrix.

Table 2. Digestive recovery of CQAs in Gom-chi according to various cooking methods

\begin{tabular}{cccccc}
\hline \multirow{2}{*}{ Cooking methods } & \multicolumn{5}{c}{$\begin{array}{c}\text { Amount of CQAs }(\boldsymbol{\mu g} / \mathbf{g} \text { fresh weight) } \\
\text { (Bioaccessibility, \%) }\end{array}$} \\
\cline { 2 - 6 } Fresh & 5-CQA & 4-CQA & 3-CQA & 3,4-di-CQA & Total CQAs \\
\hline \multirow{2}{*}{ Blanching } & $33.38 \pm 2.65$ & $344.67 \pm 18.14$ & $14.85 \pm 1.33$ & $51.96 \pm 0.95$ & $444.85 \pm 21.71^{\mathrm{a}}$ \\
3 min & $(24.41)$ & $(16.07)$ & $(88.34)$ & $(12.58)$ & $(16.42)$ \\
Blanching & $3.01 \pm 0.18$ & $21.58 \pm 1.98$ & $1.89 \pm 0.11$ & $5.38 \pm 0.13$ & $33.85 \pm 2.00^{\mathrm{b}}$ \\
5 min & $(44.02)$ & $(17.59)$ & $(84.75)$ & $(9.66)$ & $(17.64)$ \\
Pan frying & $1.10 \pm 0.78$ & $10.89 \pm 1.38$ & $0.61 \pm 0.07$ & $2.38 \pm 0.18$ & $14.99 \pm 2.41^{\mathrm{e}}$ \\
3 min & $(11.70)$ & $(13.67)$ & $(25.31)$ & $(9.78)$ & $(13.29)$ \\
Pan frying & $2.17 \pm 0.13$ & $14.41 \pm 0.42$ & $0.77 \pm 0.06$ & $2.58 \pm 0.08$ & $19.93 \pm 0.59^{\mathrm{d}}$ \\
$\mathbf{5}$ min & $(15.75)$ & $(17.81)$ & $(16.89)$ & $(4.17)$ & $(12.43)$ \\
Microwaving & N.D & N.D & N.D & N.D & N.D \\
2 min & (N.A) & (N.A) & (N.A) & $($ N.A) & (N.A) \\
Microwaving & N.D & N.D & N.D & N.D & N.D \\
3 min & (N.A) & (N.A) & (N.A) & (N.A) & (N.A) \\
\hline
\end{tabular}

*N.D (Not detected): Quantitative amount of CQAs below the limit of detection. N.A (Not applicable) *Different letters indicate significant difference among cooking methods at $\mathrm{p}<0.05$.

\subsection{Effect of Cooking Methods on Bioaccessibility of CQAs in Gom-Chi}

The bioaccessibility of total CQAs (\%) in Gom-chi according to various cooking methods ranged from 12.43 to $17.64 \%$ was accessed (Table 2). Among CQAs in fresh Gom-chi, 3-CQA recovered the most after in vitro digestion model system (88.34\%) followed by 5-CQA (24.41\%), 4-CQA (16.07), and 3,4-di-CQA (12.58\%). The bioaccessibility of total CQAs was significantly greater in Gom-chi blanched for $3 \mathrm{~min}$ than fresh and other cooked ones $(\mathrm{p}<0.05)$. As the cooking time increased, the bioaccessibility of total CQAs were reduced 0.82 and 0.94 times in blanching and pan frying, respectively. However, the bioaccessibility of total CQAs in Gom-chi pan fried for $3 \mathrm{~min}$ was slightly but not significantly higher than pan frying for $5 \mathrm{~min}$. To our knowledge, there are limited previous digestive stability data on absorption of various CQAs in humans. The studies that were done on stability of 3-CQA measured the digestive recovery of this compound after incubation with human gastric juice. The stability of 3-CQA expressed as percentages was $101 \%$ and $99 \%$ after 30 min and 120 min incubation with human gastric juice, respectively (Olthof et al., 2001). This indicated that the 3-CQA was more stable in the human digestive system. Therefore, the bioaccessibility of 3-CQA from fresh Gom-chi was higher than other CQAs. A previous study (Tagliazucchi et al., 2012) observed that the bioaccessibility of total CQAs in $50 \%$ coffee in water was approximately $65 \%$. Compared to current study, CQAs from coffee was more bioaccessible than that from Gom-chi. The decrease in the bioaccessibility of CQAs from Gom-chi after digestion may be due to degradation. A previous study (Tagliazucchi et al., 2012) reported that a general degradation of 3-CQA takes place during the in vitro digestion process of capers and sea fennel. Also, Hornero-Méndez et al., (Hornero-Méndez \& Mínguez-Mosquera, 2007) revealed that the bioaccessibility of carrot crude contained the carotenoids was reduced from $27 \%$ to $13 \%$ after cooking (heating at $100{ }^{\circ} \mathrm{C}$ in a water bath for $15 \mathrm{~min}$ ). Through these results, we can conclude that cooking procedures affect bioaccessibility of bioactive components from the food matrix.

\section{Conclusions}

In conclusion, cooking has a significant role in the content, oxygen radical absorbance capacity, and bioaccessibility of CQAs from Gom-chi. Fresh Gom-chi contained the highest amount of each CQA, and the 
scavenging ability of peroxy free radicals. Among various cooking methods, microwaved Gom-chi had the most CQAs, but barely digestible in simulating the human GI tract. Among cooking methods, Gom-chi with blanching for $3 \mathrm{~min}$ provided the greatest bioaccessibility of total CQA. Therefore, blanching for 3 min may be considered a useful preparation method for enhancing absorption and biological functions of CQAs from Gom-chi.

\section{Acknowledgements}

This work was supported by the National Research Foundation of Korea (NRF) grant funded by the Korea government (MEST) (No.2014R1A2A2A01007627)

\section{References}

Delonga, K., \& Mazor, S. (2009). Polyphenolic content and composition and antioxidative activity of different cocoa liquors. Czech journal of food sciences, 27(5), 330-337.

Farah, A., De Paulis, T., Trugo, L. C., \& Martin, P. R. (2005). Effect of roasting on the formation of chlorogenic acid lactones in coffee. Journal of Agricultural and Food Chemistry, 53(5), 1505-1513. http://dx.doi.org/10.1021/jf048701t

Hornero-Mendez, D., \& Minguez-Mosquera, M. I. (2007). Bioaccessibility of carotenes from carrots: Effect of cooking and addition of oil. Innovative Food Science \& Emerging Technologies, 8(3), 407-412. http://dx.doi.org/10.1016/j.ifset.2007.03.014

Huang, D., Ou, B., Hampsch-Woodill, M., Flanagan, J. A., \& Prior, R. L. (2002). High-throughput assay of oxygen radical absorbance capacity (ORAC) using a multichannel liquid handling system coupled with a microplate fluorescence reader in 96-well format. Journal of agricultural and food chemistry, 50(16), 4437-4444. http://dx.doi.org/10.1021/jf0201529

Kim, S., Kang, S., \& Um, B. (2010). Extraction conditions of radical scavenging caffeoylquinic acids from Gomchui (Ligularia fischeri) tea. Journal of The Korean Society of Food Science and Nutrition, 39(3), 399-405.

Kim, S. M., Jeon, J.-S., Kang, S. W., JunG, Y.-J., LY, L. N., \& Um, B.-H. (2012). Content of antioxidative caffeoylquinic acid derivatives in field-grown Ligularia fischeri (Ledeb.) Turcz and responses to sunlight. Journal of agricultural and food chemistry, 60(22), 5597-5603. http://dx.doi.org/10.1021/jf300976y

Koop, W. (2000). Effects of Microwave Appliances on Food and Humans. The Price-Pottenger Nutrition Foundation, 16(2).

Lee, B.-I., Nugroho, A., Bachri, M. S., Choi, J., Lee, K. R., Choir, J. S., ... Park, H.-J. (2010). Anti-ulcerogenic effect and HPLC analysis of the caffeoylquinic acid-rich extract from Ligularia stenocephala. Biological and Pharmaceutical Bulletin, 33(3), 493-497. http://dx.doi.org/10.1248/bpb.33.493

Lee, H. R., Cho, S. D., Lee, W. K., Kim, G. H., \& Shim, S. M. (2013). Digestive recovery of sulfur-methyl-1-methionine and its bioaccessibility in Kimchi cabbages using a simulated in vitro digestion model system. Journal of the Science of Food and Agriculture, 94(1), 109-112. http://dx.doi.org/10.1002/jsfa.6205

Miglio, C., Chiavaro, E., Visconti, A., Fogliano, V., \& Pellegrini, N. (2007). Effects of different cooking methods on nutritional and physicochemical characteristics of selected vegetables. Journal of agricultural and food chemistry, 56(1), 139-147. http://dx.doi.org/10.1021/jf072304b

Natella, F., Belelli, F., Ramberti, A., \& Scaccini, C. (2010). Microwave and traditional cooking methods: effect of cooking on antioxidant capacity and phenolic compounds content of seven vegetables. Journal of Food Biochemistry, 34(4), 796-810.

Olthof, M. R., Hollman, P. C., \& Katan, M. B. (2001). Chlorogenic acid and caffeic acid are absorbed in humans. The Journal of nutrition, 131(1), 66-71.

Pellegrini, N., Chiavaro, E., Gardana, C., Mazzeo, T., Contino, D., Gallo, M., ... Porrini, M. (2010). Effect of different cooking methods on color, phytochemical concentration, and antioxidant capacity of raw and frozen brassica vegetables. Journal of Agricultural and Food Chemistry, 58(7), 4310-4321. http://dx.doi.org/10.1021/jf904306r

Shang, Y. F., Kim, S. M., Song, D. G., Pan, C. H., Lee, W. J., \& Um, B. H. (2010). Isolation and identification of antioxidant compounds from Ligularia fischeri. Journal of Food Science, 75(6), C530-C535. http://dx.doi.org/10.1111/j.1750-3841.2010.01714.x 
Shim, S. M. (2012). Changes in profiling of phenolic compounds, antioxidative effect and total phenolic content in Similax china under in vitro physiological condition. Journal of Food Biochemistry, 36(6), 748-755. http://dx.doi.org/10.1111/j.1745-4514.2011.00589.x

Tagliazucchi, D., Helal, A., Verzelloni, E., \& Conte, A. (2012). The Type and Concentration of Milk Increase the in Vitro Bioaccessibility of Coffee Chlorogenic Acids. Journal of Agricultural and Food Chemistry, 60(44), 11056-11064. http://dx.doi.org/10.1021/jf302694a

Yang, U.-J., Yoon, S.-R., Chung, J.-H., Kim, Y. J., Park, K.-H., Park, T.-S., \& Shim, S.-M. (2012). Water spinach (Ipomoea aquatic Forsk.) reduced the absorption of heavy metals in an in vitro bio-mimicking model system. Food and Chemical Toxicology, 50(10), 3862-3866. http://dx.doi.org/10.1016/j.fct.2012.07.020

\section{Copyrights}

Copyright for this article is retained by the author(s), with first publication rights granted to the journal.

This is an open-access article distributed under the terms and conditions of the Creative Commons Attribution license (http://creativecommons.org/licenses/by/3.0/). 